\title{
SOLUTION OF SCHRÖDINGER EQUATION FOR POSCHL-TELLER PLUS SCARF NON-CENTRAL POTENTIAL USING SUPERSYMMETRY QUANTUM MECHANICS APROACH
}

\author{
Antomi Saregar \\ Physics Education Department, Lampung Raden intan Institute, Indonesia; E-mail : tomi_presma@yahoo.co.id
}

\begin{abstract}
In this paper, we show that the exact energy eigenvalues and eigenfunctions of the Schrödinger equation for charged particles moving in certain class of noncentral potentials can be easily calculated analytically in a simple and elegant manner by using Supersymmetric method (SUSYQM). We discuss the Poschl-Teller plus Scarf non-central potential systems. Then, by operating the lowering operator we get the ground state wave function, and the excited state wave functions are obtained by operating raising operator repeatedly. The energy eigenvalue is expressed in the closed form obtained using the shape invariant properties. The results are in exact agreement with other methods.
\end{abstract}

Keyword: supersymmetry, non-central potentials, poschl teller plus scarf.

\section{INTRODUCTION}

One of the important work in theoretical physics is to obtain exact solution of the Schrödinger equation for special potentials (Ballentine, 1999). It is well known that exact solution of Schrödinger equation are only possible for certain cases. The exact solution of Schrödinger equation for a class of noncentral potentials already studied in quantum chemistry (Gonul and Kocak, 2005), With the advent of supersymmetric quantum mechanics SUSYQM (Gonul and Zorba, 2000), and the idea of shape invariance (Gendenshtein1983), study of potential problems in non-relativistic quantum theory has received renewed interest. SUSYQM allows one to determine eigenvalues and eigenstates of known analytically solvable potentials using algebra operator formalism without ever having to solve the Schrödinger differential equation by standard series method. However, the operator method has so far been applied only to one dimensional and spherically symmetric theree dimensional problems. Supersymmetri is, by definition (Ranabir at all, 1988), a symmetry between fermions and boson. A supersymmetric field theoretical model consists of a set of quantum fields and of a lagrangian for them which exhibit such a symmetry. The Lagrangian determines, through the action principle, the equations of motion and hence the dynamical behaviour of the particle.

Recently, some authors have investigated the energy spectra and eigenfunction with Non-central potential (Meyur at all, 2009), Trigonometric PoschlTeller plus Rosen-Morse using SUSY (Antomi at all, 2013), Hulthén plus Manning-Rosen potential, and Scarf poential plus Poschl-Teller using NU (Cari at all, 2012). In this paper, we investigate the energy eigenvalues and eigenfunction of the Poschl-Teller plus Scarf non-central potential system using SUSYQM aproach. The trigonometric Scarf potential is also called as generalized Poschl-Teller potential (Xu at all, 2010), The trigonometric Poschl-Teller play the essential roles in electrodynamics interatomic and intermolecular forces and can be used to describe molecular vibrations. Some of these trigonometric potential are exactly solvable or quasi exactly solvable and their bound state solutions have been reported(El Kineni, 2001). 
REVIEW OF FORMULA FOR SUPERSYMMETRY QUANTUM MECHANICS

\section{A.Supersymmetry Quantum Mechanics (SUSY QM)}

Witten defined the algebra of a supersymmetry quantum system, there are super charge operators $Q_{i}$ which commute with the Hamiltonian $H_{s s}$ [21]

$$
\left[Q_{i}, H_{s s}\right]=0 \text { with, } i=1,2,3, \ldots \mathrm{N}
$$

and they obey to algebra $\left\{Q_{i}, Q_{j}\right\}=\delta_{i j} H_{s s}$

with $H_{s s}$ is called Supersymmetric Hamiltonian. Witten stated that the simplest quantum mechanical system has $\mathrm{N}=2$, it was later shown that the case where $\mathrm{N}=1$, if it is supersymmetric, it is equivalent to an $\mathrm{N}=2$ supersymmetric quantum system (Ranabir, 1988), In the case where $\mathrm{N}=2$ we can define,

$$
\begin{aligned}
& Q_{1}=\frac{1}{\sqrt{2}}\left(\sigma_{1} \frac{p}{\sqrt{2 m}}+\sigma_{2} \phi(x)\right) \text { and } \\
& Q_{2}=\frac{1}{\sqrt{2}}\left(\sigma_{2} \frac{p}{\sqrt{2 m}}+\sigma_{1} \phi(x)\right)
\end{aligned}
$$

Here the $\sigma_{i}$ are the usual Pauli spin matrices, and $p=-1 \hbar \frac{\partial}{\partial x}$ is the usual momentum operator. For example two component, we shall write $H_{s s}$ as $H_{ \pm}$. using equation (1) and (2) we get,

$$
\begin{aligned}
& H_{s s}=\left(\begin{array}{cc}
-\frac{\hbar^{2}}{2 m} \frac{d^{2}}{d x^{2}}+\frac{\hbar}{\sqrt{2 m}} \frac{d \phi(x)}{d x}+\phi^{2}(x) & 0 \\
0 & -\frac{\hbar^{2}}{2 m} \frac{d^{2}}{d x^{2}}-\frac{\hbar}{\sqrt{2 m}} \frac{d \phi(x)}{d x}+\phi^{2}(x)
\end{array}\right) \\
& =\left(\begin{array}{cc}
H_{+} & 0 \\
0 & H_{-}
\end{array}\right)
\end{aligned}
$$

with,

$$
\begin{aligned}
H_{-} & =-\frac{\hbar^{2}}{2 m} \frac{d^{2}}{d x^{2}}+V_{-}(x) \text { with } \\
V_{-}(x) & =\phi^{2}(x)-\frac{\hbar}{\sqrt{2 m}} \phi^{\prime}(x)
\end{aligned}
$$

and,

$$
\begin{aligned}
& H_{+}=-\frac{\hbar^{2}}{2 m} \frac{d^{2}}{d x^{2}}+V_{+}(x), \text { with } \\
& V_{+}(x)=\phi^{2}(x)+\frac{\hbar}{\sqrt{2 m}} \phi^{\prime}(x)
\end{aligned}
$$

with $H_{-}$and $H_{+}$, is defined as supersymmetry partner in the Hamiltonian. $V_{-}(x)$ and $V_{+}(x)$ are the supersymmetry partner each other.

Thus, solving equation (4a) and (4b), Hamiltonian equation can be faktorizated,

$$
\begin{aligned}
H_{+}(x) & =A^{+} A, \text { and } H_{-}(x)=A A^{+} \\
\text {were, } A^{+} & =-\frac{\hbar}{\sqrt{2 m}} \frac{d}{d x}+\phi(x) \text { and } \\
A & =\frac{\hbar}{\sqrt{2 m}} \frac{d}{d x}+\phi(x)
\end{aligned}
$$

with, $A^{+}$as raising operator, and $A$ as lowering operator.

\section{Shape Invariance}

Gendenshtein [6] discovered another symmetry which if the supersymmetric system satisfies it will be an exactly solvable system, this symmetry is known as shape invariance. If our potential satisfies shape invariance we can readily write down its bound state spectrum, and with the help of the charge operators we can find the bound state wave functions. It turned out that all the potentials which were known to be exactly solvable until then have the shape invariance symmetry. If the supersymmetric partner potentials have the same dependence on $x$ but differ in a parameter, in such a way that they are related to each other by a change of of that parameter, then they are said to be shape invariant. Gendenshtein stated this condition in this way,

$$
\begin{aligned}
& V_{+}\left(x ; a_{j}\right)=V_{-}\left(x ; a_{j+1}\right)+R\left(a_{j+1}\right) \\
& \text { with, } V_{+}\left(x ; a_{j}\right)=\phi^{2}\left(x ; a_{j}\right)+\frac{\hbar}{\sqrt{2 m}} \phi^{\prime}\left(x ; a_{j}\right) \\
& V_{-}\left(x ; a_{j}\right)=\phi^{2}\left(x ; a_{j}\right)-\frac{\hbar}{\sqrt{2 m}} \phi^{\prime}\left(x ; a_{j}\right)
\end{aligned}
$$

where $j=0,1,2, .$. , and $a$ is a parameter in our original potential whose ground state energy is zero. $a_{j+1}=f\left(a_{j}\right)$ where $f$ is assumed to be an arbitrary function for the time being. The remainder $R\left(a_{j}\right)$ can be dependent on the parametrization variable $a$ 
but never on $x$. In this case $V_{-}$is said to be shape invariant, and we can readily find its spectrum, take a look at $H$,

$$
H=H_{-}+E_{0}=-\frac{\hbar^{2}}{2 m} \frac{d^{2}}{d x^{2}}+V_{-}\left(x ; a_{0}\right)+E_{0}
$$

Acoording to equation.(9) a further equation is obtained between $V_{+}(x)$ and $V_{-}(x)$ we get,

$$
V(x)=V_{-}\left(x ; a_{0}\right)+E_{0}=\phi^{2}\left(x ; a_{0}\right)-\frac{\hbar}{\sqrt{2 m}} \phi^{\prime}\left(x ; a_{0}\right)+E_{0}
$$

where $V(x)$ is often stated os effective potential $V_{\text {eff }}$. While $\phi(x)$ is determined hypothetically based on the shape of effective potential from the associated system.

The hamiltonian equation can be generalized,

$$
H_{k}=-\frac{\hbar^{2}}{2 m} \frac{d^{2}}{d x^{2}}+V_{-}\left(x ; a_{k}\right)+\sum_{i=1}^{k} R\left(a_{i}\right),
$$

dengan $k=0,1,2, \ldots$

By comparing equation (8) and (9), it is found that $E_{0}=\sum_{i=1}^{k} R\left(a_{i}\right)$. So that, in eigen energy spectra, the value of $H_{-}$, can be generalized as follows,

$$
E_{n}^{(-)}=\sum_{k=1}^{n} R\left(a_{k}\right)
$$

Furthermore, we get the total energy spectra,

$$
E_{n}=E_{n}^{(-)}+E_{0}
$$

with $E_{0}$ as ground state energy in a

Hamiltonian lowering partner potential.

Based on the characteristics of lowering operator, then the equation of ground state wave function can be obtained from the following equation,

$$
A \psi_{0}{ }^{(-)}=0
$$

Meanwhile, the excited wave function, one and so forth $\psi_{n}^{-}\left(x ; a_{0}\right)$ can be obtained by using raising operator and ground state wave function $\psi_{0}^{-}\left(x ; a_{0}\right)$. In general, the equation of wave function can be stated as follow,

$$
\psi_{n}^{(-)}\left(x ; a_{0}\right) \approx A^{+}\left(x ; a_{0}\right) \psi_{n-1}^{(-)}\left(x ; a_{1}\right)
$$

SOLUTION OF SCHRÖDINGER EQUATION FOR POSCHL-TELLER PLUS TRIGONOMETRIC SCARF NON-CENTRAL POTENTIAL USING SUPERSYMMETRY

Schrödinger equation Poschl-Teller plus Scarf Non-central potential is the potentials present simulataneusly in the quantum system. This non-central potential is expressed as,

$V(r, \theta)=\frac{\hbar^{2}}{2 m \alpha^{2}}\left(\frac{a(a-1)}{\sin ^{2}\left(\frac{r}{\alpha}\right)}+\frac{b(b-1)}{\cos ^{2}\left(\frac{r}{\alpha}\right)}\right)+\frac{\hbar^{2}}{2 m r^{2}}\left(\frac{b^{2}+a(a-1)}{\sin ^{2} \theta}-\frac{2 b\left(a-\frac{1}{2}\right) \cos \theta}{\sin ^{2} \theta}\right)$

The three dimensional Schrödinger equation for Poschl-Teller plus trigonometric Scarf non-central potential is written as,

$-\frac{\hbar^{2}}{2 m}\left[\frac{1}{r^{2}} \frac{\partial}{\partial r}\left(r^{2} \frac{\partial \psi}{\partial r}\right)+\frac{1}{r^{2} \sin \theta} \frac{\partial}{\partial \theta}\left(\sin \theta \frac{\partial \psi}{\partial \theta}\right)+\frac{1}{r^{2} \sin ^{2} \theta} \frac{\partial^{2} \psi}{\partial \varphi^{2}}\right]$ $+\frac{\hbar^{2}}{2 m \alpha^{2}}\left(\frac{a(a-1)}{\sin ^{2}\left(\frac{r}{\alpha}\right)}+\frac{b(b-1)}{\cos ^{2}\left(\frac{r}{\alpha}\right)}\right) \psi++\frac{\hbar^{2}}{2 m r^{2}}\left(\frac{b^{2}+a(a-1)}{\sin ^{2} \theta}-\frac{2 b\left(a-\frac{1}{2}\right) \cos \theta}{\sin ^{2} \theta}\right) \psi=E \psi$

If equation (17) multiplied by factor, ( $\left.-\frac{2 m r^{2}}{\hbar^{2}}\right)$, and the result is solve using separation variable method since the noncentral potential is separable. By setting $\psi(r, \theta, \varphi)=R(r) P(\theta) \Phi(\varphi)$, with $R P \Phi\left(\psi(r, \theta, \varphi)=\frac{X(r) Q(\theta) e^{i m \varphi}}{r \sqrt{\sin \theta}}, \quad\right.$ we obtain, $\left[\frac{1}{R} \frac{\partial}{\partial r}\left(r^{2} \frac{\partial R}{\partial r}\right)-\frac{r^{2}}{\alpha^{2}}\left(\frac{a(a-1)}{\sin ^{2}\left(\frac{r}{\alpha}\right)}+\frac{b(b-1)}{\cos ^{2}\left(\frac{r}{\alpha}\right)}\right)+\frac{2 m r^{2}}{\hbar^{2}} E\right]$ $+\left[\frac{1}{P \sin \theta} \frac{\partial}{\partial \theta}\left(\sin \theta \frac{\partial P}{\partial \theta}\right)+\frac{1}{\Phi \sin ^{2} \theta} \frac{\partial^{2} \Phi}{\partial \varphi^{2}}-\left(\frac{b^{2}+a(a-1)}{\sin ^{2} \theta}-\frac{2 b\left(a-\frac{1}{2}\right) \cos \theta}{\sin ^{2} \theta}\right)\right]=0$

from equation (18) we obtain radial and angular Schrödinger equation as,

$\frac{1}{R} \frac{\partial}{\partial r}\left(r^{2} \frac{\partial R}{\partial r}\right)-\frac{r^{2}}{\alpha^{2}}\left(\frac{a(a-1)}{\sin ^{2}\left(\frac{r}{\alpha}\right)}+\frac{b(b-1)}{\cos ^{2}\left(\frac{r}{\alpha}\right)}\right)+\frac{2 m r^{2}}{\hbar^{2}} E$
$=\left(\frac{b^{2}+a(a-1)}{\sin ^{2} \theta}-\frac{2 b\left(a-\frac{1}{2}\right) \cos \theta}{\sin ^{2} \theta}\right)-\frac{1}{P \sin \theta} \frac{\partial}{\partial \theta}\left(\sin \theta \frac{\partial P}{\partial \theta}\right)-\frac{1}{\Phi \sin ^{2} \theta} \frac{\partial^{2} \Phi}{\partial \varphi^{2}}=\ell(\ell+1)$

with $l(l+1)$ is constanta variabel separable, where $\ell$ as orbital momentum number. 
From equation (19) we get radial and angular wave function Schrödinger equation with single variable as following,

$\frac{1}{R} \frac{\partial}{\partial r}\left(r^{2} \frac{\partial R}{\partial r}\right)-\frac{r^{2}}{\alpha^{2}}\left(\frac{a(a-1)}{\sin ^{2}\left(\frac{r}{\alpha}\right)}+\frac{b(b-1)}{\cos ^{2}\left(\frac{r}{\alpha}\right)}\right)+\frac{2 m r^{2}}{\hbar^{2}} E=\ell(\ell+1)$

or equation (20) multiplied by $\left(\frac{R}{r}\right)$, with $R(r)=\frac{\chi(r)}{r}$, so using symple algebra, we get,

$\frac{\partial^{2} \chi}{\partial r^{2}}-\frac{1}{\alpha^{2}}\left(\frac{a(a-1)}{\sin ^{2}\left(\frac{r}{\alpha}\right)}+\frac{b(b-1)}{\cos ^{2}\left(\frac{r}{\alpha}\right)}\right) \chi-\frac{\ell(\ell+1)}{r^{2}} X=-\frac{2 m}{\hbar^{2}} E \chi$

and than, for solve radial Schrödinger equation, we use approximation for centrifugal term,

$\frac{1}{r^{2}} \cong \frac{1}{\alpha^{2}}\left(d_{0}+\frac{1}{\sin ^{2}\left(\frac{r}{\alpha}\right)}\right) \quad$ for $\quad \frac{r}{\alpha}<<1, \quad$ with $d_{0}=\frac{1}{12}, \in^{2}=-\frac{2 m}{\hbar^{2}} E$, we get,

$\frac{\partial^{2} \chi}{\partial r^{2}}-\frac{1}{\alpha^{2}}\left(\frac{a(a-1)}{\sin ^{2}\left(\frac{r}{\alpha}\right)}+\frac{b(b-1)}{\cos ^{2}\left(\frac{r}{\alpha}\right)}\right) \chi-\ell(\ell+1) \frac{1}{\alpha^{2}}\left(d_{0}+\frac{1}{\sin ^{2}\left(\frac{r}{\alpha}\right)}\right) \chi=\epsilon^{2} \chi$

from equation (22) simplied by $\left(-\frac{\hbar^{2}}{2 m}\right)$ we get radial Schrödinger equation,

$-\frac{\hbar^{2}}{2 m} \frac{\partial^{2} \chi}{\partial r^{2}}+\frac{\hbar^{2}}{2 m \alpha^{2}}\left(\frac{a(a-1)}{\sin ^{2}\left(\frac{r}{\alpha}\right)}+\frac{b(b-1)}{\cos ^{2}\left(\frac{r}{\alpha}\right)}\right) \chi+\frac{\hbar^{2}}{2 m \alpha^{2}} \ell(\ell+1) d_{0} \chi=-\frac{\hbar^{2}}{2 m} \epsilon^{2} \chi$

and we have the angular and Schrödinger equation as,

$\left(\frac{a(a-1)}{\sin ^{2}\left(\frac{r}{\alpha}\right)}+\frac{b(b-1)}{\cos ^{2}\left(\frac{r}{\alpha}\right)}\right)-\frac{1}{P \sin \theta} \frac{\partial}{\partial \theta}\left(\sin \theta \frac{\partial P}{\partial \theta}\right)-\frac{1}{\Phi \sin ^{2} \theta} \frac{\partial^{2} \Phi}{\partial \varphi^{2}}=\ell(\ell+1)$

and we have set $\frac{1}{\Phi} \frac{\partial^{2} \Phi}{\partial \varphi^{2}}=-m^{2}$ that give azimuthalwave function as

$$
\Phi=\sqrt{\frac{1}{2 \pi}} e^{i m \varphi}, m=0, \pm 1, \pm 2, \ldots
$$

equation (24) will be,

$\left(\frac{b^{2}+a(a-1)}{\sin ^{2} \theta}-\frac{2 b\left(a-\frac{1}{2}\right) \cos \theta}{\sin ^{2} \theta}\right)-\frac{1}{P \sin \theta} \frac{\partial}{\partial \theta}\left(\sin \theta \frac{\partial P}{\partial \theta}\right)+\frac{m^{2}}{\sin ^{2} \theta}=\ell(\ell+1)$

with $m^{2}$ as variable separation and we get angular Schrödinger equation one dimentional, $-\frac{\hbar^{2}}{2 m} \frac{d^{2}}{d \theta^{2}}+\frac{\hbar^{2}}{2 m}\left(\frac{b^{2}+a(a-1)+m^{2}-\frac{1}{4}}{\sin ^{2} \theta}-\frac{2 b\left(a-\frac{1}{2}\right) \cos \theta}{\sin ^{2} \theta}\right) H=\frac{\hbar^{2}}{2 m}\left(\ell(\ell+1)+\frac{1}{4}\right) H$

The solution of Radial Scrodinger equation Poschl Teller plus trigonometric Scarf potential

Factor $\mathrm{R}$ in equation (20) is defined as wave function $\psi$, then the Schrödinger equation for Poschl-Teller plus Scarf noncentral potential in radial with the assumption of $\epsilon^{\prime}=-\frac{\hbar^{2}}{2 m} \epsilon^{2}$ can be rewritten as follow,

$-\frac{\hbar^{2}}{2 m} \frac{\partial^{2} \chi}{\partial r^{2}}+\frac{\hbar^{2}}{2 m \alpha^{2}}\left(\frac{a(a-1)+\ell(\ell+1)}{\sin ^{2}\left(\frac{r}{\alpha}\right)}+\frac{b(b-1)}{\cos ^{2}\left(\frac{r}{\alpha}\right)}\right) \chi+\frac{\hbar^{2}}{2 m \alpha^{2}} \ell(\ell+1) d_{0} \chi=\epsilon^{\prime} \chi$

Based on equation (28), the effective potential of radial SE PoschlTeller plus trigonometric Scarf non-central potential can be rewritten as follow,

$V_{\text {eff }}=\frac{\hbar^{2}}{2 m \alpha^{2}}\left(\frac{a(a-1)+\ell(\ell+1)}{\sin ^{2}\left(\frac{r}{\alpha}\right)}+\frac{b(b-1)}{\cos ^{2}\left(\frac{r}{\alpha}\right)}\right)+\frac{\hbar^{2}}{2 m \alpha^{2}} \ell(\ell+1) d_{0}$

or,

$V_{\text {eff }}=\frac{\hbar^{2}}{2 m \alpha^{2}}\left(\frac{a^{\prime}\left(a^{\prime}-1\right)}{\sin ^{2}\left(\frac{r}{\alpha}\right)}+\frac{b(b-1)}{\cos ^{2}\left(\frac{r}{\alpha}\right)}\right)+\frac{\hbar^{2}}{2 m \alpha^{2}} \ell(\ell+1) d_{0}$

with $a^{\prime}=\sqrt{a(a-1)+\ell(\ell+1)+\frac{1}{4}}+\frac{1}{2}$

By inserting effective potential in equation (30) into equation (10), its obtained

$\phi^{2}(x)-\frac{\hbar}{\sqrt{2 m}} \phi^{\prime}(x)=\frac{\hbar^{2}}{2 m \alpha^{2}}\left(\frac{a^{\prime}\left(a^{\prime}-1\right)}{\sin ^{2}\left(\frac{r}{\alpha}\right)}+\frac{b(b-1)}{\cos ^{2}\left(\frac{r}{\alpha}\right)}\right)+\frac{\hbar^{2}}{2 m \alpha^{2}} \ell(\ell+1) d_{0}-\epsilon$

By using incisive hypothesis, it is assumed that superpotential in equation (30) is,

$\phi(x)=A \tan \left(\frac{r}{\alpha}\right)+B \cot \left(\frac{r}{\alpha}\right)$

Where $\mathrm{A}$ and $\mathrm{B}$ are indefinite constantans that will be calculated. From equation (32), we can determine the value of $\phi^{\prime}(x)$ and $\phi^{2}(x)$, then the result is distributed into equation (31), then the following is obtained,

$$
\begin{aligned}
& \frac{A^{2}}{\cos ^{2}\left(\frac{r}{\alpha}\right)}-A^{2}+\frac{B^{2}}{\sin ^{2}\left(\frac{r}{\alpha}\right)}-B^{2}+2 A B-\frac{\hbar}{\alpha \sqrt{2 m}}\left(\frac{A}{\cos ^{2}\left(\frac{r}{\alpha}\right)}-\frac{B}{\sin ^{2}\left(\frac{r}{\alpha}\right)}\right) \\
& \quad=\frac{\hbar^{2}}{2 m \alpha^{2}}\left(\frac{a^{\prime}\left(a^{\prime}-1\right)}{\sin ^{2}\left(\frac{r}{\alpha}\right)}+\frac{b(b-1)}{\cos ^{2}\left(\frac{r}{\alpha}\right)}\right)+\frac{\hbar^{2}}{2 m \alpha^{2}} \ell(\ell+1) d_{0}-\epsilon
\end{aligned}
$$


By analysing the similar concept between left flank and right flank, from equation (33), it is obtained,

$$
\begin{aligned}
& \left(A^{2}-\frac{\hbar}{\alpha \sqrt{2 m}} A\right) \frac{1}{\cos ^{2}\left(\frac{r}{\alpha}\right)}=\frac{\hbar^{2}}{2 m \alpha^{2}}(b(b-1)) \frac{1}{\cos ^{2}\left(\frac{r}{\alpha}\right)} ; \\
& \left(B^{2}+\frac{\hbar}{\alpha \sqrt{2 m}} B\right) \frac{1}{\sin ^{2}\left(\frac{r}{\alpha}\right)}=-\frac{\hbar^{2}}{2 m \alpha^{2}}\left(\frac{a^{\prime}\left(a^{\prime}-1\right)}{\sin ^{2}\left(\frac{r}{\alpha}\right)}\right) \\
& \text {; } \\
& \text { and } A^{2}+B^{2}-2 A B+\frac{\hbar^{2}}{2 m \alpha^{2}} \ell(\ell+1) d_{0}=\in=E_{0}
\end{aligned}
$$

from the three equation in equation (34), it is obtained,

$$
\begin{aligned}
& A_{+}=\frac{\hbar}{\alpha \sqrt{2 m}} b \text { or } A_{-}=-\frac{\hbar}{\alpha \sqrt{2 m}}(b-1) \\
& B_{+}=\frac{\hbar}{\alpha \sqrt{2 m}}\left(a^{\prime}-1\right) \text { or } B_{-}=-\frac{\hbar}{\alpha \sqrt{2 m}} a^{\prime} \\
& E_{\mathrm{O}}=\frac{\hbar}{\alpha \sqrt{2 m}}\left(\left(b+a^{\prime}\right)^{2}+\ell(\ell+1) d_{\mathrm{O}}\right)
\end{aligned}
$$

The value of $\mathrm{A}$ and $\mathrm{B}$ are determined in certain way so the value of $E_{0}^{(-)}$is equal to zero, so,

$$
\phi(x)=-\frac{\hbar}{\alpha \sqrt{2 m}} b \tan \left(\frac{r}{\alpha}\right)-\frac{\hbar}{\alpha \sqrt{2 m}} a^{\prime} \cot \left(\frac{r}{\alpha}\right)
$$

By using equation. (6) and (36), we get

$$
A^{+}=-\frac{\hbar}{\sqrt{2 m}} \frac{d}{d r}+\phi(r)=-\frac{\hbar}{\sqrt{2 m}} \frac{d}{d r}+\frac{\hbar}{\alpha \sqrt{2 m}} b \tan \left(\frac{r}{\alpha}\right)-\frac{\hbar}{\alpha \sqrt{2 m}} a^{\prime} \cot \left(\frac{r}{\alpha}\right)
$$

and

$$
A=\frac{\hbar}{\sqrt{2 m}} \frac{d}{d r}+\phi(r)=\frac{\hbar}{\sqrt{2 m}} \frac{d}{d r}+\frac{\hbar}{\alpha \sqrt{2 m}} b \tan \left(\frac{r}{\alpha}\right)-\frac{\hbar}{\alpha \sqrt{2 m}} a^{\prime} \cot \left(\frac{r}{\alpha}\right)
$$

The ground state wave function can be obtained from equation (14) and (38), which are,

$$
\left\{\frac{\hbar}{\sqrt{2 m}} \frac{d}{d r}+\frac{\hbar}{\alpha \sqrt{2 m}} b \tan \left(\frac{r}{\alpha}\right)-\frac{\hbar}{\alpha \sqrt{2 m}} a^{\prime} \cot \left(\frac{r}{\alpha}\right)\right\} \psi_{0}^{-}=0
$$

Then the ground state wave function of rosen more potential is as follow,

$$
\begin{aligned}
\int \frac{d \psi_{0}^{-}}{\psi_{0}^{-}}\left(r, a_{0}\right)= & a^{\prime} \int \cot \left(\frac{r}{\alpha}\right) d\left(\frac{r}{\alpha}\right)-b \int \tan \left(\frac{r}{\alpha}\right) d\left(\frac{r}{\alpha}\right) \\
\ln \psi_{0}^{-}\left(r, a_{0}\right)= & \left\{a^{\prime} \ln \sin \left(\frac{r}{\alpha}\right)-b \ln \cos \left(\frac{r}{\alpha}\right)\right\}+C \\
& -K \ln (\csc \alpha r-\cot \alpha r)\}+C \\
\psi_{0}^{-}\left(r, a_{0}\right)= & C\left(\cos \left(\frac{r}{\alpha}\right)\right)^{b}\left(\sin \left(\frac{r}{\alpha}\right)\right)^{a^{\prime}}
\end{aligned}
$$

By using equation (15) we can obtain excited wave function on the first level as follow,

$\psi_{1}^{(-)}\left(r ; a_{0}\right) \approx A^{+}\left(x ; a_{0}\right) \psi_{0}^{(-)}\left(r ; a_{1}\right)$ where $a_{0}=a^{\prime}=a_{1}=a^{\prime}+1, \quad \ldots \ldots, a_{n}=a^{\prime}+n$ is the independent parametre to variable " $r$ ". By inserting the value of the parametre to equation (39) and (37) and by using equation (40), the following we get,

$$
\begin{gathered}
\psi_{1}^{(-)}\left(r ; a_{0}\right) \approx\left(-\frac{\hbar}{\sqrt{2 m}} \frac{d}{d r}+\frac{\hbar}{\alpha \sqrt{2 m}} b \tan \left(\frac{r}{\alpha}\right)-\frac{\hbar}{\alpha \sqrt{2 m}} a^{\prime} \cot \left(\frac{r}{\alpha}\right)\right) \\
\left\{C\left(\cos \left(\frac{r}{\alpha}\right)\right)^{(b+1)}\left(\sin \left(\frac{r}{\alpha}\right)\right)^{\left(a^{\prime}+1\right)}\right\} \\
\approx-\frac{\hbar C}{\alpha \sqrt{2 m}}\left(-(b+1)\left(\cos \left(\frac{r}{\alpha}\right)\right)^{b}\left(\sin \left(\frac{r}{\alpha}\right)\right)^{\left(a^{\prime}+2\right)}+\left(a^{\prime}+1\right)\left(\cos \left(\frac{r}{\alpha}\right)\right)^{(b+2)}\left(\sin \left(\frac{r}{\alpha}\right)\right)^{a^{\prime}}\right) \\
+\left(\frac{\hbar}{\alpha \sqrt{2 m}} b \tan \left(\frac{r}{\alpha}\right)-\frac{\hbar}{\alpha \sqrt{2 m}} a^{\prime} \cot \left(\frac{r}{\alpha}\right)\right) \\
\left.\approx C\left(\cos \left(\frac{r}{\alpha}\right)\right)^{(b+1)}\left(\sin \left(\frac{r}{\alpha}\right)\right)^{\left(a^{\prime}+1\right)}\right\} \\
\frac{\hbar}{\alpha \sqrt{2 m}}\left((2 b+1)\left(\sin \left(\frac{r}{\alpha}\right)\right)^{2}-\left(2 a^{\prime}+1\right)\left(\cos \left(\frac{r}{\alpha}\right)\right)^{2}\right) \\
\left\{C\left(\cos \left(\frac{r}{\alpha}\right)\right)^{b}\left(\sin \left(\frac{r}{\alpha}\right)\right)^{a^{\prime}}\right\}
\end{gathered}
$$

The breakdown in equation (41) can be continued to find wave function $\psi_{2}^{(-)}\left(r ; a_{0}\right), \psi_{3}^{(-)}\left(x ; a_{0}\right), \ldots$ and so on.

The determination of the potential partner which have shape invariant, by using equation (8a) and (8b) results, $V_{-}\left(r, a_{0}\right)=\frac{\hbar^{2}}{2 m \alpha^{2}}\left(\frac{b(b-1)}{\cos ^{2}\left(\frac{r}{\alpha}\right)}+\frac{a^{\prime}\left(a^{\prime}-1\right)}{\sin ^{2}\left(\frac{r}{\alpha}\right)}\right)-\frac{\hbar^{2}}{2 m \alpha^{2}}\left(a^{\prime}+b\right)^{2}$ and,

$V_{+}\left(r, a_{0}\right)=\frac{\hbar^{2}}{2 m \alpha^{2}}\left(\frac{a^{\prime}\left(a^{\prime}+1\right)}{\cos ^{2}\left(\frac{r}{\alpha}\right)}+\frac{b(b+1)}{\sin ^{2}\left(\frac{r}{\alpha}\right)}\right)-\frac{\hbar^{2}}{2 m \alpha^{2}}\left(a^{\prime}+b\right)^{2}$

If we have choose parameters $a_{0}=v^{\prime}, a_{1}=$ $v^{\prime}+1, a_{2}=v^{\prime}+2, \ldots$ then $V_{-}\left(r, a_{1}\right)$

Obtained if on equ. (42) the value of $v^{\prime}$, changed into $v^{\prime}+1$, i.e

$V_{-}\left(r, a_{1}\right)=\frac{\hbar^{2}}{2 m \alpha^{2}}\left(\frac{a^{\prime}\left(a^{\prime}+1\right)}{\cos ^{2}\left(\frac{r}{\alpha}\right)}+\frac{b(b+1)}{\sin ^{2}\left(\frac{r}{\alpha}\right)}\right)-\frac{\hbar^{2}}{2 m \alpha^{2}}\left(a^{\prime}+b+2\right)^{2}$

From those equ. (42b) and (43) can be seen that $\mathrm{V}_{+}\left(\mathrm{r}, \mathrm{a}_{0}\right)$ have similar shape with $V_{-}\left(r, a_{1}\right)$, and with using shape invariance relation on equ. (8) obtained $\mathrm{R}\left(\mathrm{a}_{1}\right)$ i.e,, 


$$
R\left(a_{1}\right)=V_{+}\left(r, a_{0}\right)-V_{-}\left(r, a_{1}\right)=\frac{\hbar^{2}}{2 m \alpha^{2}}\left(\left(a^{\prime}+b+2\right)^{2}-\left(a^{\prime}+b\right)^{2}\right)
$$

We repeat the step as on the determination of equ. (48) by using the steps equ. (42a), (42b), and (43), to obtain equation $V_{+}\left(r, a_{1}\right)$ and $V_{-}\left(r, a_{2}\right)$, so obtained,

$V_{+}\left(r, a_{1}\right)=\frac{\hbar^{2}}{2 m \alpha^{2}}\left(\frac{\left(a^{\prime}+1\right)\left(a^{\prime}+2\right)}{\cos ^{2}\left(\frac{r}{\alpha}\right)}+\frac{(b+1)(b+2)}{\sin ^{2}\left(\frac{r}{\alpha}\right)}\right)-\frac{\hbar^{2}}{2 m \alpha^{2}}\left(a^{\prime}+b+2\right)^{2}$

$V_{-}\left(r, a_{2}\right)=\frac{\hbar^{2}}{2 m \alpha^{2}}\left(\frac{\left(a^{\prime}+1\right)\left(a^{\prime}+2\right)}{\cos ^{2}\left(\frac{r}{\alpha}\right)}+\frac{(b+1)(b+2)}{\sin ^{2}\left(\frac{r}{\alpha}\right)}\right)-\frac{\hbar^{2}}{2 m \alpha^{2}}\left(a^{\prime}+b+4\right)^{2}$

From equ. (45a) and (45b) so obtained,

$R\left(a_{2}\right)=V_{+}\left(r, a_{1}\right)-V_{-}\left(r, a_{2}\right)=\frac{\hbar^{2}}{2 m \alpha^{2}}\left(\left(a^{\prime}+b+4\right)^{2}-\left(a^{\prime}+b+2\right)^{2}\right)$

Then, the determination steps on equ. (44) or equ. (46) above are repeated until parameters heading to $\mathrm{n}, a_{n}$ to determinate $R\left(a_{n}\right)$ and finally obtained,

$$
E_{n}^{(-)}=\sum_{k=1}^{n} R\left(a_{k}\right)=\frac{\hbar^{2}}{2 m \alpha^{2}}\left(\left(a^{\prime}+b+2 n\right)^{2}-\left(a^{\prime}+b\right)^{2}\right)
$$

If equ. (47) and equ. (36c) incorporated to equation (13) obtained energy spectrum for Poschl-Teller system i.e.,

$E_{n}=E_{n}^{(-)}+E_{0}=\frac{\hbar^{2}}{2 m \alpha^{2}}\left(\left(a^{\prime}+b+2 n\right)^{2}+\ell(\ell+1) d_{0}\right)(48)$ with, $\quad E_{n}=\in^{\prime}, \quad$ and $a^{\prime}=\sqrt{a^{\prime}\left(a^{\prime}+1\right)+\ell(\ell+1)+\frac{1}{4}}+\frac{1}{2}$, so equ. (48) can be rewritten energy solution of Schrödinger equation for Poschl-Teller plus Scarf noncentral potential as,

$$
\begin{aligned}
& -\frac{\hbar^{2}}{2 m} \in^{2}=E_{n} \\
& -\frac{\hbar^{2}}{2 m}\left(-\frac{2 m}{\hbar^{2}} E\right)=\frac{\hbar^{2}}{2 m \alpha^{2}}\left(\left(a^{\prime}+b+2 n\right)^{2}+\ell(\ell+1) d_{0}\right) \\
& E_{n r}=\frac{\hbar^{2}}{2 m \alpha^{2}}\left(\left(a^{\prime}+b+2 n\right)^{2}+\ell(\ell+1) d_{0}\right)
\end{aligned}
$$

Equation (49) showed the process of energy spectra of Poschl-Teller plus Scarf noncentral potential, with,

$\hbar$ : planck constants,

$m$ : mass of particle

$a$ and $b:$ constants potential depth,

$n$ : principe quatum numbers, $n=1,2,3 \ldots$

$n_{r}$ : radial quantum numbers, $n_{r}=0,1,2 \ldots$ $l$ : orbital quantum numbers (the value same with polar wave function solving) $l=0,1,2 \ldots n-1$.

The solution of angular Schrödinger equation Poschl-Teller plus trigonometric Scarf non-central noncentral potential.

To ease the solution of angular Schrödinger Equation, i.e.,

$$
\frac{\hbar^{2}}{2 m}\left(\ell(\ell+1)+\frac{1}{4}\right) H=E H
$$

If equ. (50) incorporated to equ. (28) so angular Schrödinger equation PoschlTeller plus Scarf non-central potential chanced into,

$$
-\frac{\hbar^{2}}{2 m} \frac{d^{2} H}{d \theta^{2}}+\frac{\hbar^{2}}{2 m}\left(\frac{b^{2}+a(a-1)+m^{2}-\frac{1}{4}}{\sin ^{2} \theta}-\frac{2 b\left(a-\frac{1}{2}\right) \cos \theta}{\sin ^{2} \theta}\right) H=E H
$$

Based on equ. (51), effective potential of angular Poschl-Teller plus trigonometric Scarf non-central potential describe as,

$V_{e f f}=\frac{\hbar^{2}}{2 m}\left(\frac{b^{2}+a(a-1)+m^{2}-\frac{1}{4}}{\sin ^{2} \theta}-\frac{2 b\left(a-\frac{1}{2}\right) \cos \theta}{\sin ^{2} \theta}\right)$

if, $a^{\prime}=\sqrt{b^{2}+a(a-1)+m^{2}}+\frac{1}{2}$, we get

$$
V_{\text {eff }}=\frac{\hbar^{2}}{2 m}\left(\frac{a^{\prime}\left(a^{\prime}-1\right)}{\sin ^{2} \theta}-\frac{2 b\left(a-\frac{1}{2}\right) \cos \theta}{\sin ^{2} \theta}\right)
$$

According to the form of those effective potential equations, then superpotential equation of Angular PoschlTeller plus trigonometric Scarf non-central potential can be describe as,

$$
\phi(\theta)=A \cot \theta+B \csc \theta
$$

where $\mathrm{A}$ and $\mathrm{B}$ are unstable constant that will be counted. From equ. (54), determinated value of $\phi_{0}^{\prime}(x)$ and $\phi_{0}^{2}(x)$, thus the results are subtituted into equation (6), obtained relation,

$$
\begin{aligned}
& \frac{A^{2}}{\sin ^{2} \theta}-A^{2}+\frac{B^{2}}{\sin ^{2} \theta}+2 A B \frac{\cos \theta}{\sin ^{2} \theta}+\frac{\hbar}{\sqrt{2 m}} \frac{A}{\sin ^{2} \theta} \\
& =\frac{\hbar^{2}}{2 m}\left(\frac{a^{\prime}\left(a^{\prime}-1\right)}{\sin ^{2} \theta}-\frac{2 b\left(a-\frac{1}{2}\right) \cos \theta}{\sin ^{2} \theta}\right)+\frac{\hbar^{2}}{2 m} \ell(\ell+1) d_{0}-\epsilon
\end{aligned}
$$


By using in common concept of coefficient between left and right internode, so that from equation (55), value is obtained,

$$
\begin{aligned}
& \left(A^{2}+B^{2}+\frac{\hbar}{\sqrt{2 m}} A\right) \frac{1}{\sin ^{2} \theta}=\frac{\hbar^{2}}{2 m}\left(a^{\prime}\left(a^{\prime}-1\right)\right) \frac{1}{\sin ^{2} \theta} ; \\
& \left(2 A B+\frac{\hbar}{\sqrt{2 m}} B\right) \frac{\cos \theta}{\sin ^{2} \theta}=-\frac{\hbar^{2}}{2 m}\left(\frac{2 b\left(a-\frac{1}{2}\right) \cos \theta}{\sin ^{2} \theta}\right) ; \\
& \text { and } A^{2}+\frac{\hbar^{2}}{2 m} \ell(\ell+1) d_{0}=\in=E_{0}
\end{aligned}
$$

from those third equation on equ. (56) is obtained,

$$
A_{+}=\frac{\hbar}{\sqrt{2 m}}\left(\sqrt{\frac{\left(a^{\prime}-\frac{1}{2}\right)^{2}-\sqrt{\left(\left(a^{\prime}-\frac{1}{2}\right)^{2}\right)^{2}-4\left(b\left(a-\frac{1}{2}\right)\right)^{2}}}{2}}-\frac{1}{2}\right)
$$

atau

$$
A_{-}=-\frac{\hbar}{\sqrt{2 m}}\left(\sqrt{\frac{\left(a^{\prime}-\frac{1}{2}\right)^{2}-\sqrt{\left(\left(a^{\prime}-\frac{1}{2}\right)^{2}\right)^{2}-4\left(b\left(a-\frac{1}{2}\right)\right)^{2}}}{2}}+\frac{1}{2}\right)
$$

$$
B=\frac{\hbar}{\sqrt{2 m}}\left(\frac{b\left(a-\frac{1}{2}\right)}{\sqrt{\frac{\left(a^{\prime}-\frac{1}{2}\right)^{2}-\sqrt{\left(\left(a^{\prime}-\frac{1}{2}\right)^{2}\right)^{2}-4\left(b\left(a-\frac{1}{2}\right)\right)^{2}}}{2}}}\right)
$$

$E_{0}=\frac{\hbar^{2}}{2 m}\left(\sqrt{\frac{\left(a^{\prime}-\frac{1}{2}\right)^{2}-\sqrt{\left(\left(a^{\prime}-\frac{1}{2}\right)^{2}\right)^{2}-4\left(b\left(a-\frac{1}{2}\right)\right)^{2}}}{2}}+\frac{1}{2}\right)+\frac{\hbar^{2}}{2 m} \ell(\ell+1) d_{0}$

$\mathrm{A}$ and $\mathrm{B}$ value are chosen so that $E_{0}^{(-)}$value is zero.

By using equ. (8a) and (8b) are obtained,

$$
\begin{gathered}
\phi(r)=-\frac{\hbar}{\sqrt{2 m}}\left(\sqrt{\frac{\left(a^{\prime}-\frac{1}{2}\right)^{2}-\sqrt{\left(\left(a^{\prime}-\frac{1}{2}\right)^{2}\right)^{2}-4\left(b\left(a-\frac{1}{2}\right)\right)^{2}}}{2}}+\frac{1}{2}\right) \cot \theta \\
+\frac{\hbar}{\sqrt{2 m}}\left(\frac{b\left(a-\frac{1}{2}\right)}{\sqrt{\frac{\left(a^{\prime}-\frac{1}{2}\right)^{2}-\sqrt{\left.\left(a^{\prime}-\frac{1}{2}\right)^{2}\right)^{2}-4\left(b\left(a-\frac{1}{2}\right)\right)^{2}}}{2}}} \csc \theta\right) \\
\phi(r)=-\frac{\hbar}{\sqrt{2 m}}\left(M+\frac{1}{2}\right) \cot \theta+\frac{\hbar}{\sqrt{2 m}} K \csc \theta
\end{gathered}
$$

with, $\quad M=\sqrt{\frac{\left(a^{\prime}-\frac{1}{2}\right)^{2}-\sqrt{\left(\left(a^{\prime}-\frac{1}{2}\right)^{2}\right)^{2}-4\left(b\left(a-\frac{1}{2}\right)\right)^{2}}}{2}}$ and

$$
K=\frac{b\left(a-\frac{1}{2}\right)}{\sqrt{\frac{\left(a^{\prime}-\frac{1}{2}\right)^{2}-\sqrt{\left(\left(a^{\prime}-\frac{1}{2}\right)^{2}\right)^{2}-4\left(b\left(a-\frac{1}{2}\right)\right)^{2}}}{2}}}
$$

$$
\begin{aligned}
& V_{-}\left(\theta, a_{0}\right)=\frac{\hbar^{2}}{2 m} \frac{\left(\left(M+\frac{1}{2}\right)^{2}+K^{2}-\left(M+\frac{1}{2}\right)\right)}{\sin ^{2} \theta} \\
& -\frac{\hbar^{2}}{2 m} K\left(2\left(M+\frac{1}{2}\right)-1\right) \frac{\cos \theta}{\sin ^{2} \theta}-\frac{\hbar^{2}}{2 m}\left(M+\frac{1}{2}\right)^{2} \\
& V_{+}\left(\theta, a_{0}\right)=\frac{\hbar^{2}}{2 m} \frac{\left(\left(M+\frac{1}{2}\right)^{2}+K^{2}+\left(M+\frac{1}{2}\right)\right)}{\sin ^{2} \theta} \\
& -\frac{\hbar^{2}}{2 m} K\left(2\left(M+\frac{1}{2}\right)+1\right) \frac{\cos \theta}{\sin ^{2} \theta}-\frac{\hbar^{2}}{2 m}\left(M+\frac{1}{2}\right)^{2}
\end{aligned}
$$

From those two equations (59a) and (59b) is obtained $a_{0}=M ; \quad a_{1}=M^{\prime}+1 ; \ldots$

$$
\begin{gathered}
V_{-}\left(\theta, a_{1}\right)=\frac{\hbar^{2}}{2 m} \frac{\left((M+1)^{2}+K^{2}-\frac{1}{4}\right)}{\sin ^{2} \theta} \\
-\frac{\hbar^{2}}{2 m} K(2(M+1)) \frac{\cos \theta}{\sin ^{2} \theta}-\frac{\hbar^{2}}{2 m}\left(M+\frac{3}{2}\right)^{2}
\end{gathered}
$$

From those two equ. (59b) and (60) can be seen that $\mathrm{V}_{+}\left(\theta, \mathrm{a}_{0}\right)$ have the same form with $V_{-}\left(\theta, a_{1} b_{1}\right)$, and by using shape invariance relation on equ. (8), is obtained $\mathrm{R}\left(\mathrm{a}_{1}\right)$ i.e., $R\left(a_{1}\right)=V_{+}\left(\theta ; a_{0}\right)-V_{-}\left(\theta ; a_{1}\right)$

$$
=\frac{\hbar^{2}}{2 m}\left(\left(M+\frac{3}{2}\right)^{2}-\left(M+\frac{1}{2}\right)^{2}\right)
$$

We repeated the step as on determination of equ. (61) with using steps equ. (59), and equ. (60) to obtain $V_{+}\left(\theta, a_{1}\right)$ and $V_{-}\left(\theta, a_{2}\right)$ equations, so obtained,

$$
\begin{aligned}
V_{+}\left(\theta, a_{1}\right)= & \frac{\hbar^{2}}{2 m} \frac{\left(M^{2}+4 M+\frac{15}{4}+K^{2}\right)}{\sin ^{2} \theta} \\
& -\frac{\hbar^{2}}{2 m} K(2(M+2)) \frac{\cos \theta}{\sin ^{2} \theta}-\frac{\hbar^{2}}{2 m}\left(M+\frac{3}{2}\right)^{2}
\end{aligned}
$$

$$
\begin{aligned}
& V_{-}\left(\theta, a_{2}\right)=\frac{\hbar^{2}}{2 m} \frac{\left(M^{2}+4 M+\frac{15}{4}+K^{2}\right)}{\sin ^{2} \theta} \\
& -\frac{\hbar^{2}}{2 m} K(2(M+2)) \frac{\cos \theta}{\sin ^{2} \theta}-\frac{\hbar^{2}}{2 m}\left(M+\frac{5}{2}\right)^{2} \\
& (62 \mathrm{~b})
\end{aligned}
$$

By repeated the step from equ. (62a) to (62b) we often,

$$
R\left(a_{2}\right)=V_{+}\left(\theta, a_{1}\right)-V_{-}\left(\theta, a_{2}\right)=\frac{\hbar^{2}}{2 m}\left(\left(M+\frac{5}{2}\right)^{2}-\left(M+\frac{3}{2}\right)^{2}\right)
$$




$$
\begin{aligned}
& R\left(a_{n}\right)=V_{+}\left(\theta ; a_{n-1}\right)-V_{-}\left(\theta ; a_{n}\right) \\
& =\frac{\hbar^{2}}{2 m}\left(M+\frac{1}{2}+n\right)^{2}-\frac{\hbar^{2}}{2 m}\left(b+a^{\prime}+(n-1)\right)^{2} .
\end{aligned}
$$

Then determination steps on equ. (61) or equ. (63) on above are repeated until parameters heading to $\mathrm{n}, a_{n} b_{n}$ to deteminate $R\left(a_{n} b_{n}\right)$ as on equ. (64) and finally obtained the order of energy parameters that described,

$$
E_{n}^{(-)}=\sum_{k=1}^{n} R\left(a_{k}\right)=\frac{\hbar^{2}}{2 m}\left(\left(M+\frac{1}{2}+n\right)^{2}-\left(M+\frac{1}{2}\right)^{2}\right)
$$

If equ. (65) and equ. (57c) are inserted into equation (13) we obtain energy spectrum of Poschl-Teller plus trigonometric Scarf noncentral system so,

$$
\begin{aligned}
& E_{n}=E_{n}^{(-)}+E_{0}=\frac{\hbar^{2}}{2 m}\left(\left(M+\frac{1}{2}+n\right)^{2}-\left(M+\frac{1}{2}\right)^{2}+\left(M+\frac{1}{2}\right)^{2}\right) \\
& \text { so } E_{n}=\frac{\hbar^{2}}{2 m}\left(M+\frac{1}{2}+n\right)^{2}
\end{aligned}
$$

By using the same order of energy parameters with eig

$$
\left(\ell(\ell+1)+\frac{1}{4}\right)=\left(M+\frac{1}{2}+n\right)^{2}
$$

then $\ell= \pm(M+n)$

angular quantum numbers on equation (67) is used to calculate energy spectrum potential non-central system. obtained

By using equ. (6) and (58) are

$$
\begin{aligned}
& A^{+}=-\frac{\hbar}{\sqrt{2 m}} \frac{d}{d r}-\frac{\hbar}{\sqrt{2 m}}\left(\left(M+\frac{1}{2}\right) \cot \theta-(K \csc \theta)\right) \\
& \text { and } A=\frac{\hbar}{\sqrt{2 m}} \frac{d}{d r}-\frac{\hbar}{\sqrt{2 m}}\left(\left(M+\frac{1}{2}\right) \cot \theta-(K \csc \theta)\right)(68 \mathrm{~b})
\end{aligned}
$$

By using decreasing operator on equ (68b), determinated basic wave function for angular Poschl-Teller plus trigonometric Scarf non-central potetial as follows,

$$
\begin{aligned}
& \left\{\frac{\hbar}{\sqrt{2 m}} \frac{d}{d r}-\frac{\hbar}{\sqrt{2 m}}\left(\left(M+\frac{1}{2}\right) \cot \theta-(K \csc \theta)\right)\right\} \psi_{0}^{-}=0 \\
& \left.\int \frac{d \psi_{0}^{-}}{\psi_{0}^{-}}\left(\theta, a_{0}\right)=\left(M+\frac{1}{2}\right) \int \cot \theta d \theta-K\right) \int \csc \theta d \theta \\
& \ln \psi_{0}^{-}\left(\theta, a_{0}\right)=\left\{\left(M+\frac{1}{2}\right) \ln \sin \theta\right. \\
& -K \ln (\csc \theta-\cot \theta)\}+C
\end{aligned}
$$

$$
\begin{aligned}
\psi_{0}^{-}\left(\theta, a_{0}\right)= & C(\sin \theta)^{\left(M+\frac{1}{2}\right)}\left(\frac{1}{\sin \theta}-\frac{\cos \theta}{\sin \theta}\right)^{(-K)} \\
& =C(\sin \theta)^{\left(M+\kappa+\frac{1}{2}\right)}(1-\cos \theta)^{(-K)}(69)
\end{aligned}
$$

Then, by using increasing operator on equ (68a) and basic wave function determinated first level excited wave function,

$$
\begin{aligned}
& \psi_{1}^{(-)}\left(\theta ; a_{0}\right) \approx A^{+}\left(\theta ; a_{0}\right) \psi_{0}^{(-)}\left(\theta, a_{1}\right) \\
& \psi_{1}^{(-)}\left(\theta ; a_{0}\right) \approx\left(-\frac{\hbar}{\sqrt{2 m}} \frac{d}{d r}-\frac{\hbar}{\sqrt{2 m}}\left(\left(M+\frac{1}{2}\right) \cot \theta-K \csc \theta\right)\right)\left\{C(\sin \theta)^{\left(M+\times x^{\frac{3}{2}}\right)}(1-\cos \theta)^{(-K)}\right\}
\end{aligned}
$$$$
\begin{aligned}
\approx & \left(-C \frac{\hbar}{\sqrt{2 m}}\left(\frac{d}{d r}(\sin \theta)^{\left(M+K+\frac{3}{2}\right)}(1-\cos \theta)^{(-K)}+(\sin \theta)^{\left(1+\times+\frac{3}{2}\right)} \frac{d}{d r}(1-\cos \theta)^{(-K)}\right)\right) \\
& -\frac{\hbar}{\sqrt{2 m}}\left[\left(\left(M+\frac{1}{2}\right) \cot \theta-K \csc \theta\right)\left(C(\sin \theta)^{\left(M+K+\frac{3}{2}\right)}(1-\cos \theta)^{(-K)}\right)\right]
\end{aligned}
$$

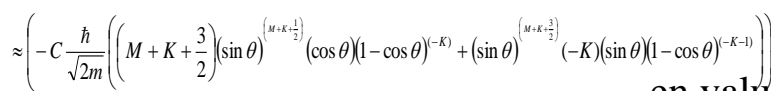
$-\frac{\hbar}{\sqrt{2 m}}\left(\left(M+\frac{1}{2}\right) \cot \theta-K \csc \theta\right)\left\{C(\sin \theta)^{\left(M+K \frac{3}{2}\right)}(1-\cos \theta)^{(-K)}\right\}$

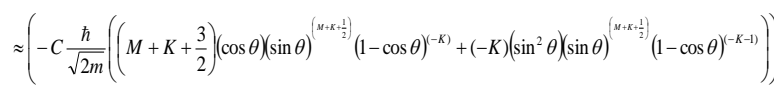

$$
\begin{aligned}
& -\left\{\frac{\hbar}{\sqrt{2 m}}((M+1 / 2) \cot \theta-K \csc \theta)\left(C(\sin \theta)^{\left(M+K+\frac{3}{2}\right)}(1-\cos \theta)^{(-K)}\right)\right\}
\end{aligned}
$$$$
\begin{aligned}
& \approx\left(-\frac{\hbar}{\sqrt{2 m}}\left(\left(M+K+\frac{3}{2}\right) \cos \theta+(-K)\left(\sin ^{2} \theta\right) \frac{1}{(1-\cos \theta)}\right)-\frac{\hbar}{\sqrt{2 m}}\left(\left(M+\frac{1}{2}\right) \frac{\cos \theta}{\sin \theta}(\sin \theta)-\frac{K}{\sin \theta}(\sin \theta)\right)\right) \\
& \quad\left\{C(\sin \theta)^{\left(M+\kappa+\frac{1}{2}\right)}(1-\cos \theta)^{(-K)}\right\} \\
& \approx\left(-\frac{\hbar}{\sqrt{2 m}}\left(\left(M+K+\frac{3}{2}\right)(\cos \theta)-K \frac{\left(\sin ^{2} \theta\right)}{(1-\cos \theta)}\right)-\frac{\hbar}{\sqrt{2 m}}\left(\left(M+\frac{1}{2}\right)(\cos \theta)-K\right)\right) \\
& \quad\left\{C(\sin \theta)^{\left(M+K+\frac{1}{2}\right)}(1-\cos \theta)^{(-K)}\right\} \\
& \approx\left(-\frac{\hbar}{\sqrt{2 m}}\left((2 M+K+2)(\cos \theta)-K \frac{\left(\sin ^{2} \theta\right)}{(1-\cos \theta)}-K\right)\right)
\end{aligned}
$$

$$
\left\{C(\sin \theta)^{\left(M+\kappa+\frac{1}{2}\right)}(1-\cos \theta)^{(-K)}\right\}
$$

To determinate excited wave function above can be done as on determination of first level excited wave function as follows,

$$
\psi_{2}{ }^{(-)}\left(\theta ; a_{0}\right), \psi_{3}^{(-)}\left(\theta ; a_{0}\right), \text { and so }
$$

on. 
Therefore obtained wave function level that is wanted.

Since $Q_{n}^{(-)}\left(x ; a_{n}\right)=\psi_{n}^{(-)}\left(x ; a_{n}\right)$, so we get,

$$
P=\frac{\psi_{n}{ }^{(-)}\left(\theta ; a_{0}\right)}{\sqrt{\sin \theta}}
$$

$$
\begin{aligned}
P=\psi_{1}^{(-)}\left(\theta ; a_{0}\right) \approx & \left(-\frac{\hbar}{\sqrt{2 m}}\left((2 M+K+2)(\cos \theta)-K \frac{\left(\sin ^{2} \theta\right)}{(1-\cos \theta)}-K\right)\right) \\
& \left\{C(\sin \theta)^{(M+K)}(1-\cos \theta)^{(-K)}\right\}(72)
\end{aligned}
$$

With,

$$
\begin{gathered}
M=\sqrt{\frac{\left(a^{\prime}-\frac{1}{2}\right)^{2}-\sqrt{\left(\left(a^{\prime}-\frac{1}{2}\right)^{2}\right)^{2}-4\left(b\left(a-\frac{1}{2}\right)\right)^{2}}}{2}} ; \\
K=\frac{b\left(a-\frac{1}{2}\right)}{\sqrt{\frac{\left(a^{\prime}-\frac{1}{2}\right)^{2}-\sqrt{\left(\left(a^{\prime}-\frac{1}{2}\right)^{2}\right)^{2}-4\left(b\left(a-\frac{1}{2}\right)\right)^{2}}}{2}}} ;
\end{gathered}
$$

and, $a^{\prime}=\sqrt{b^{2}+a(a-1)+m^{2}}+\frac{1}{2}$

Furthermore, the result of each calculation from $1^{\text {st }}$ and $2^{\text {nd }}$ excited wave function, with the value $n_{l}, \mathrm{~m}$, a, and $\mathrm{b}$, which is from table 2 , at the same time altogether with the visualisation of polar wave function shown on table 2 , below,

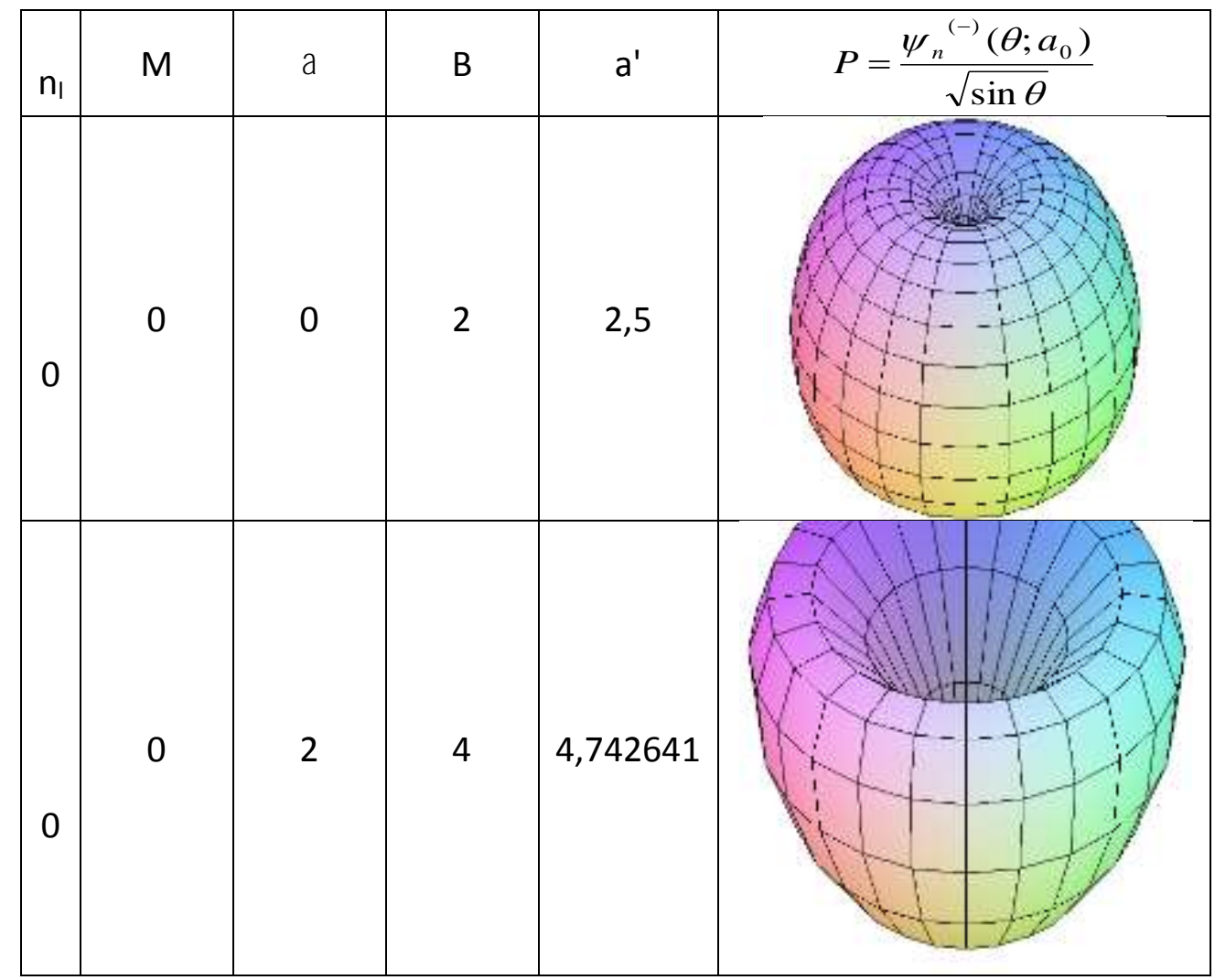




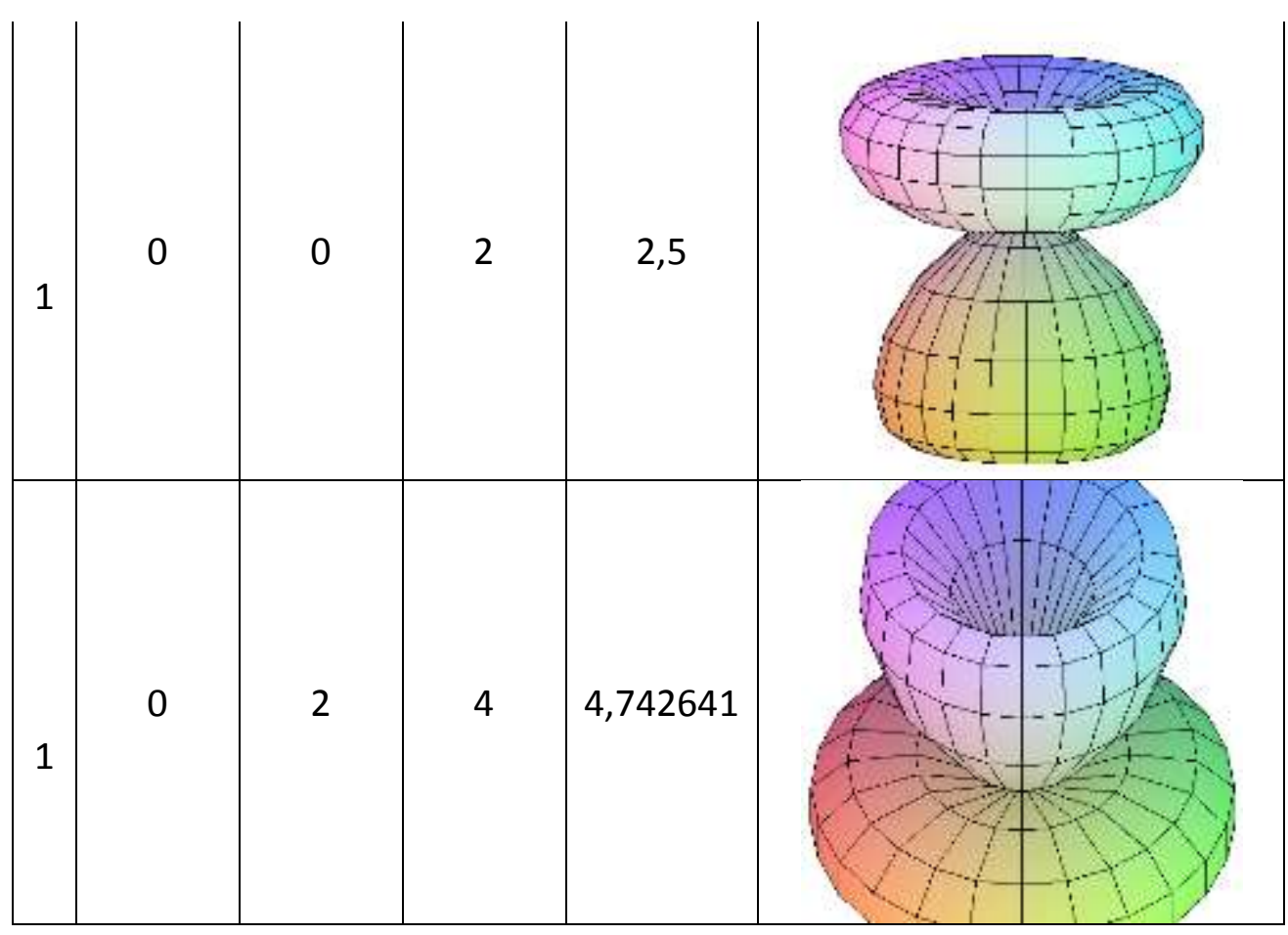

\section{CONCLUSION}

Based on the describtion, proved that Poschl-Teller plus trigonometric Scarf noncentral potential for group of shape invariance potential can be solved with Supersimmetric method.

\section{REFERENCE}

Ballentine, 1999, Quantum mechanics, Simon Fraser University

Gonul.B and Zorba.I, Phys. Lett. A Vol 269 (2000) 83-88, Supersymmetric Solution of Non-Central Potetials, Department of Engineering Physics, Gaziantep University, Turkey

Gonul. B and Kocak. M, arXiv : quantph/0409085v3], Systemic Search of Exactly Solvable Non-Central Potentials, Department of Engineering Physics, Gaziantep University, Turkey,(2005)

F. Cooper, A. Khare, U. Sukhatme, Phys. Rep. 251_1995.
267, (1984)

L Gendenshtein L. E, Derivation of exact spectra of the schrodinger equation by means of supersymmetry.JETP Letters,38(6):356, (1983)

Ranabir Dutt, Avinash Khare, and Uday P. Sukhatme. Supersymmetry, shape invariance, and exactly solvable potentials. American Journal of Physics, 56(2):163-168, (1988).

M.F. Sohnius, Introducing Supersymmetry (Cambridge CB3 9EW, NHC: England, (1985)

Witten E., Dynamical Breaking of Supersymmetry, Nucl. Phys. B185,513554, (1981)

Suparmi, Desertation, Semiclassical SUSY approace in Quantum Mechanics, Department of Physics, Suny at Albany. USA, (1992)

Antomi S, .Suparmi, Cari, Hatma Y. Research Inventy International Journal Engineering and Science. Analysis of Energy Spectra and Wave Function of Trigonometric Poschl-Teller plus Rosen Morse Non-Central Potentials 
Using Supersymmetric Quantum Mechanics Approach, Department of Physics, Sebelas Maret Universitas Indonesia, (2013)

Cari, Suparmi, IOSR Journal of Applied Physics. Approximate Solution of Schrodinger equation for Trigonometric Scarf with the PoschlTeller Non-Central Potentials Using NU Method, Physics Department, Sebelas Maret Universitas. Indonesia, (2012)

S. Meyur, S. Debnath. Eigen spectra for Woods-Saxon plus Rosen-Morse potential, Lat. Am. J. Phys. Educ. Vol. 4, No. 3, Sept. (2010)

Y. Xu, S. He and C.S. Jia, Approximate analytical solutions of the KleinGordon equation with theP"oschlTeller potential including the centrifugal term, Phys. Scr. 81 (2010) 045001.

A.H. El Kineni and M. Daoud, Coherent States a la Klaude-Perelomov for the Poschl-Teller potentials,Phys. Lett. A 283 (2001) 291-299 